\title{
Fast-food consumers in Singapore: demographic profile, diet quality and weight status
}

\author{
Clare Whitton ${ }^{1, *}, \mathrm{Yi} \mathrm{Ma}^{1}$, Amber Carla Bastian${ }^{2}$, Mei Fen Chan ${ }^{1}$ and Ling Chew ${ }^{3}$ \\ ${ }^{1}$ Research and Evaluation Department, Research and Strategic Planning Division, Health Promotion Board, \\ 3 Second Hospital Avenue, Singapore 168937: ${ }^{2}$ Centre of Excellence (Nutrition), Adult Health Division, Health \\ Promotion Board, Singapore: ${ }^{3}$ Research and Strategic Planning Division, Health Promotion Board, Singapore
}

Submitted 19 December 2012: Final revision received 15 May 2013: Accepted 18 June 2013: First published online 2 August 2013

\begin{abstract}
Objective: To determine the demographic profile of fast-food consumers among adult Singapore residents and ascertain whether fast-food consumption frequency is associated with diet quality and weight status.

Design: A nationally representative cross-sectional survey including an FFQ and anthropometric measures. Participants were grouped based on their fast-food consumption frequency as non-consumer, occasional consumer or regular consumer, with regular defined as at least once per week.

Setting: Individuals living in the community in Singapore.

Subjects: Singapore residents ( $n$ 1627) aged $18-69$ years of Chinese, Malay and Indian ethnicity.

Results: Proportions of regular fast-food consumers were higher in younger age groups, higher income groups and middle education level groups. Mean daily energy intake was positively associated with fast-food consumption frequency (non-consumers $9636 \mathrm{~kJ}$ (2303 kcal); occasional consumers $11159 \mathrm{~kJ}$ (2667 kcal); regular consumers $13100 \mathrm{~kJ}(3131 \mathrm{kcal}) ; P$ for trend $<0 \cdot 001)$. Fast-food consumers were more likely to exceed the RDA for energy, fat and saturated fat, and less likely to meet wholegrain and fruit recommendations. Both regular consumers $(\mathrm{OR}=1 \cdot 24 ; 95 \% \mathrm{CI} 1 \cdot 03,1 \cdot 51)$ and occasional consumers (OR $=1 \cdot 52$; $95 \%$ CI $1.32,1.77)$ were more likely to have a waist:hip ratio indicating abdominal obesity. Occasional consumers were more likely to have a $\mathrm{BMI} \geq$ $23 \cdot 0 \mathrm{~kg} / \mathrm{m}^{2}(\mathrm{OR}=1 \cdot 19 ; 95 \% \mathrm{CI} 1 \cdot 04,1 \cdot 37)$, whereas regular consumers were less likely $(\mathrm{OR}=0 \cdot 76 ; 95 \% \mathrm{CI} 0 \cdot 64,0 \cdot 91)$ to have an 'at-risk' BMI.

Conclusions: Fast-food consumption is most prevalent in young adults, high income and middle education level groups. Frequent fast-food consumption in Singapore is associated with unfavourable dietary and nutrient profiles and abdominal obesity.
\end{abstract}

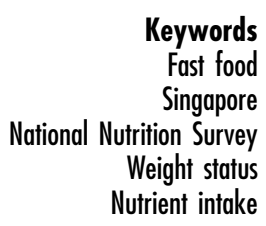

The prevalence of obesity and diet-related noncommunicable diseases is rising in Singapore. In adults, obesity prevalence rose from $6.9 \%$ in 2004 to $10 \cdot 8 \%$ in 2010, while diabetes mellitus prevalence rose from $8 \cdot 2 \%$ in 2004 to $11 \cdot 3 \%$ in $2010^{(1)}$. Energy intakes are also higher than previously; the population mean daily intake rose from $9950 \mathrm{~kJ}$ (2378 kcal) in 2004 to $10979 \mathrm{~kJ}$ (2624 kcal) in $2010^{(2)}$. Typical diets in Singapore are energy-dense. The average adult Singaporean consumes $10979 \mathrm{~kJ} / \mathrm{d}$ $(2624 \mathrm{kcal} / \mathrm{d})$, with little difference by ethnic group (Chinese $10933 \mathrm{~kJ} / \mathrm{d}(2613 \mathrm{kcal} / \mathrm{d}) ;$ Malay $11175 \mathrm{~kJ} / \mathrm{d}$ (2671 kcal/d); Indian $11058 \mathrm{~kJ} / \mathrm{d}(2643 \mathrm{kcal} / \mathrm{d}))$. Eating out is popular, since it is convenient and relatively lowpriced, with food courts and street food vendor centres being the most frequented venues. The food in these food courts is prepared by stall holders or at centralised kitchens and then transported to the retail outlets. Food courts primarily serve Chinese, Malay and Indian cuisine. Cuisines from around the region are also available, such as Korean, Japanese and Thai. More recently, many food courts have 'Western' food stalls, selling Western-type foods such as French fries and steak. Rice is the main staple in all three major ethnic groups, with noodles being popular in Chinese cuisine. Sweetened milk tea and coffee are popular. Frozen and refrigerated ready meals are not dominant parts of the Singaporean diet. In 2010, $60 \cdot 1 \%$ of Singapore residents reported usually eating out for lunch and/or dinner, compared with $47 \cdot 8 \%$ in $2004^{(2)}$. Concurrently, household food expenditure on food service has increased from $58 \%$ in $2002 / 03$ to $62 \%$ in $2007 / 08^{(3)}$. Yet despite the ease of access to a wide variety of convenient, affordable foods, the number of Western 
fast-food outlets on the island is increasing ${ }^{(4)}$; a trend seen in other countries in $\mathrm{Asia}^{(5-7)}$.

'Western' fast food tends to be energy-dense, nutrientpoor items which can undermine appetite regulation and may lead to 'passive over-consumption' ${ }^{\text {(8) }}$. Fast-food consumption has been associated with poorer diet quality, such as lower wholegrain intake ${ }^{(9)}$, lower fruit intake ${ }^{(9-11)}$, lower vegetable intake ${ }^{(9,10,12)}$ and higher intakes of fat and saturated fat ${ }^{(10)}$. Fast-food consumption has also been associated with weight status ${ }^{(11,13,14)}$ and weight gain ${ }^{(9,12,15,16)}$. In a recent analysis of a cohort of middle-aged and older Chinese Singaporeans, fastfood intake was associated with a strong risk of CHD mortality $^{(17)}$; in this cohort, $11 \%$ of participants reported consuming fast food once per week or more.

International data suggest that the proportion of fastfood consumers may vary in different segments of the population, such as younger age groups. In the $\mathrm{USA}^{(10,18)}$, Australia $^{(19)}$ and Spain ${ }^{(11)}$ fast-food consumption is highest among younger age groups. It has also been shown to be more prevalent in higher-income groups in the USA ${ }^{(10)}$, Australia $^{(19)}$ and South Africa ${ }^{(20)}$.

The aim of the present study was to investigate the demographic profile of fast-food consumers in an ethnically diverse, nationally representative sample of Singapore residents and determine whether fast-food consumption frequency in adult Singapore residents is associated with diet quality and weight status.

\section{Methods}

Data from participants aged 18-69 years who took part in the 2010 National Nutrition Survey (NNS) were used in the present analysis. NNS is a national cross-sectional survey carried out every six years to monitor food and nutrient intake at the population level. NNS 2010 participants were a sub-sample of 1773 individuals aged 18-69 years from the 2010 National Health Survey (NHS) ${ }^{(1)}$ participants. Details on sampling, data collection and data quality control can be found in the NHS 2010 report $^{(1)}$. For NNS 2010 the sampling selection matrix was stratified by gender, ethnicity and age. Malay and Indian participants were over-sampled to provide adequate numbers for statistical comparisons between ethnic groups. Interviewers underwent two days of classroom-based training before the commencement of fieldwork. Fieldwork was carried out at six locations across Singapore between March and June 2010. As part of NHS, participants' heights and weights were measured using the WHO MONICA protocol $^{(21)}$; this was converted to BMI and the Asian cutoff of $\geq 23 \mathrm{~kg} / \mathrm{m}^{2}$ for identifying individuals at moderate and high risk of obesity-related diseases was used in analysis $^{(1,22)}$. Waist and hip circumferences were also measured and Asian cut-offs for waist circumference and waist:hip ratio ${ }^{(23)}$ were used to define abdominal obesity.
Demographic variables were also collected, including age, gender, ethnicity, monthly household income and highest educational attainment. Groupings for educational attainment were 'primary or below', 'secondary/O/ N-level', 'A-level/polytechnic' and 'degree/professional qualification'. 'Primary' corresponds with the age group 6-12 years. 'Secondary/O/N-level' are secondary- or highschool qualifications, corresponding with the age group 13-17 years. 'A-level/polytechnic' are further education qualifications which are beyond the level of secondaryor high-school qualifications, but prior to university degree-level qualifications, and usually correspond with the age group 17-19 years.

A locally validated $\mathrm{FFQ}^{(24)}$ containing 182 items was administered face to face. The FFQ was semi-quantitative, including predefined serving sizes. Participants were asked how often one of these standardised servings was consumed in a 'typical' month and could answer per day, per week or per month. Food vessels such as plates, bowls and glasses were shown to participants to help them visualise 'one serving'. The nutrient database for analysing the FFQ contained the weight in grams of each of these standard servings. There was also a 'rarely/never' option for items consumed less frequently than once per month. As an introduction to the fast-food section of the FFQ, participants were told they would be asked about their fast-food consumption. The five fast-food items contained in the FFQ were: 'burgers, with beef or chicken', 'burgers, fish', 'French fries', 'pizza' and 'mashed potato with gravy'. Mashed potato with gravy is typically served at a fried chicken fast-food outlet. The term 'fast food' was not defined to participants, so reporting is based on participant perception of whether the food outlet at which the food was purchased was indeed a fast-food outlet. Data were entered into FIND (Food Information and Nutrition Database), an in-house dataentry system, merged with the corresponding nutrient profile for each FFQ item, and aggregated to produce daily food group and nutrient intakes for each participant. Questions on dietary practices such as the type of oil used in cooking were asked prior to the FFQ; answers to these questions were routed to corresponding FFQ line items in order that more accurate nutrient profiles could be assigned. In this sense there were 397 possible FFQ items.

Fast-food consumers were defined as those who reported consuming any quantity of any of the five FFQ items in the fast-food section of the questionnaire in a typical month. Participants were split into groups based on their fast-food consumption frequency: non-consumer, regular consumer and occasional consumer. 'Regular' was defined as consuming at least one serving at least once per week; this cut-off was used in other studies ${ }^{(25,26)}$ and was thought to be a fitting definition for a 'regular' behaviour. 'Occasional' was defined as consuming any quantity of fast food less than once per week but more than once per month, while a 'non-consumer' was 
defined as someone reporting rarely/never consuming fast foods. While it could not be determined whether some of the fast-food items were consumed together, it was considered likely; therefore frequencies were not summed when assigning participants to these groups. Diet quality was assessed by comparing mean daily intakes and intakes per $4184 \mathrm{~kJ}(1000 \mathrm{kcal})$ of macronutrients and selected micronutrients between the groups. Intakes per $4184 \mathrm{~kJ}$ were examined to account for the correlation of energy intake with intake of other nutrients. The odds of meeting wholegrain, fruit and vegetable guidelines were assessed, and the odds of exceeding energy, total fat and saturated fat intake recommendations were also assessed. The Health Promotion Board of Singapore recommends at least one serving of wholegrain products, two servings of fruit and two servings of vegetables per day ${ }^{(27)}$. The RDA used for energy intake recommendations was based on equations derived from a sample including Asian subjects $^{(28)}$. Fat and saturated fat are recommended to contribute no more than $30 \%$ and $10 \%$ of total energy intake, respectively ${ }^{(27)}$.

The study was conducted according to the guidelines laid down in the Declaration of Helsinki and all procedures involving human subjects were approved by the Health Promotion Board Medical and Dental Board Ethics Committee. Written informed consent was obtained from all subjects.

\section{Statistical analyses}

Weightings were applied to account for non-response, selection bias and population profile for key demographics. ANOVA with linear contrasts was used to test for trends in food and nutrient intakes across groups. Since food intake variables did not follow a normal distribution, data were first log-transformed; however, non-transformed data are presented herein for ease of interpretation. Logistic regression was used to determine the odds of meeting food-based recommendations for fruit, vegetable and wholegrain intakes, the odds of exceeding recommendations for energy, fat and saturated fat intakes, and the odds of having 'at-risk' BMI (BMI $\left.\geq 23 \cdot 0 \mathrm{~kg} / \mathrm{m}^{2}\right)$, raised waist circumference ( $\geq 90 \mathrm{~cm}$ males; $\geq 80 \mathrm{~cm}$ females) and raised waist:hip ratio ( $\geq 0.90$ males; $\geq 0.85$ females) for each of the fast-food consumption frequency groups. Age (as a continuous variable), gender, ethnicity, household income group and education level group were included in the model. Analysis was conducted in the statistical software package IBM SPSS Statistics Version 20.

\section{Results}

Of the 1773 invited participants aged 18-69 years, 1661 participants completed the FFQ. Subsequently thirty-four were excluded; fourteen due to ineligibility (e.g. belonging to an ethnic group other than Chinese, Malay or Indian, or being below 18 years of age at the time of fieldwork) and twenty due to extreme energy intakes (defined as $<2092$ or $>29288 \mathrm{~kJ} / \mathrm{d}(<500$ or $>7000 \mathrm{kcal} / \mathrm{d})$ for males and $<2092$ or $>20920 \mathrm{~kJ} / \mathrm{d} \quad(<500$ or $>5000 \mathrm{kcal} / \mathrm{d})$ for females). Therefore 1627 participants were included in the comparison of food and nutrient intakes. One hundred and sixty-seven participants had not provided either income or education level data and were not included in logistic regression for the determination of odds ratios.

\section{Demographics}

Overall $63 \%$ of participants reported consuming fast food within a typical month, and $20 \%$ did so at least once per week. Frequency of consumption differed by age, gender, ethnicity, household income and education level (Table 1). Proportions reporting consumption (either regular or occasional) decreased with age from $89 \%$ in the youngest age group (18-29 years) to $28 \%$ in the oldest (60-69 years). Conversely, proportions reporting consumption increased with monthly household income from $49 \%$ in the lowest income group to $81 \%$ in the highest, and also increased with education level, from $29 \%$ in the group with lowest education level to $79 \%$ in the group with highest. However, regular consumption was highest in the middle education level group. Proportions reporting consumption were more similar by gender and ethnicity, but were highest in males (68\%) and Malays (73\%).

\section{Food and nutrient intakes}

A positive association was observed between frequency of fast-food consumption and total food intake (Table 2). Compared with non-consumers of fast food, median food intake of occasional and regular fast-food consumers was higher by $189 \mathrm{~g}$ and $462 \mathrm{~g}$, respectively $(P<0 \cdot 001)$. This trend was not confined to a particular food group but was reflected in intakes of many food groups.

In regular fast-food consumers, mean daily energy intake was $13100 \mathrm{~kJ}$ (3131 kcal), compared with $11159 \mathrm{~kJ}$ $(2667 \mathrm{kcal})$ in occasional consumers and $9636 \mathrm{~kJ}$ ( $2303 \mathrm{kcal}$ ) in non-consumers (Table 3). Intakes of energy, fat and saturated fat in comparison to dietary guidelines were positively associated with fast-food consumption frequency ( $P$ for trend $<0 \cdot 001$ ), and in regular consumers the percentage contribution of fast food to average daily intakes of these nutrients reached a daily equivalent of $6 \cdot 1-7 \cdot 9 \%$ (Table 4 ). Carbohydrate and protein intakes per $4184 \mathrm{~kJ}$ were inversely associated with fast-food consumption frequency, whereas fat intake per $4184 \mathrm{~kJ}$ was positively associated with fast-food consumption frequency ( $P$ for trend $<0 \cdot 001$ ). Cholesterol intake per $4184 \mathrm{~kJ}$ was also positively associated with fast-food consumption frequency ( $P$ for trend $<0 \cdot 001$ ); there was a mean difference of $10 \cdot 6(95 \% \mathrm{CI} 8 \cdot 8,12 \cdot 4)$ $\mathrm{mg} / 4184 \mathrm{~kJ}$ between regular consumers of fast food and non-consumers. Intakes of micronutrients per $4184 \mathrm{~kJ}$ were inversely associated with frequency of fast-food 
Table 1 Sociodemographic and anthropometric variables by fast-food consumption status: Singapore residents ( $n$ 1627) aged 18-69 years, 2010 National Nutrition Survey

\begin{tabular}{|c|c|c|c|c|}
\hline & \multirow[b]{2}{*}{$n$} & \multicolumn{3}{|c|}{ Fast-food consumption status (\%) } \\
\hline & & $\begin{array}{l}\text { Non-consumer } \\
\quad(n 557)\end{array}$ & $\begin{array}{l}\text { Occasional consumer } \\
(n \text { 720) }\end{array}$ & $\begin{array}{l}\text { Regular consumer } \\
\qquad(n 350)\end{array}$ \\
\hline Total & 1627 & 37 & 43 & 20 \\
\hline \multicolumn{5}{|l|}{ Gender } \\
\hline Male & 808 & 32 & 45 & 23 \\
\hline Female & 819 & 42 & 41 & 17 \\
\hline \multicolumn{5}{|l|}{ Age group (years) } \\
\hline $18-29$ & 383 & 11 & 45 & 44 \\
\hline 30-39 & 390 & 19 & 55 & 26 \\
\hline $40-49$ & 403 & 38 & 50 & 12 \\
\hline $50-59$ & 283 & 60 & 30 & 10 \\
\hline $60-69$ & 168 & 72 & 26 & 2 \\
\hline \multicolumn{5}{|l|}{ Ethnicity } \\
\hline Chinese & 666 & 40 & 42 & 18 \\
\hline Malay & 491 & 27 & 45 & 28 \\
\hline Indian & 470 & 34 & 44 & 22 \\
\hline \multicolumn{5}{|l|}{ Education level ${ }^{*}$} \\
\hline Primary or below & 276 & 72 & 23 & 6 \\
\hline Secondary/O/N-level & 581 & 47 & 38 & 15 \\
\hline A-level/polytechnic & 347 & 23 & 41 & 37 \\
\hline Degree/professional qualification & 419 & 21 & 57 & 22 \\
\hline \multicolumn{5}{|l|}{ Monthly household income $(\$ S)^{\star}$} \\
\hline$<2000$ & 377 & 51 & 35 & 14 \\
\hline 2000-3999 & 482 & 36 & 45 & 19 \\
\hline $4000-5999$ & 292 & 32 & 43 & 25 \\
\hline 6000-9999 & 194 & 24 & 50 & 26 \\
\hline$\geq 10000$ & 116 & 19 & 62 & 19 \\
\hline \multicolumn{5}{|l|}{$\mathrm{BMI}\left(\mathrm{kg} / \mathrm{m}^{2}\right)$} \\
\hline$<18 \cdot 5$ & 86 & 25 & 43 & 32 \\
\hline $18 \cdot 5-22 \cdot 9$ & 517 & 38 & 39 & 22 \\
\hline $23 \cdot 0-27 \cdot 4$ & 553 & 40 & 45 & 16 \\
\hline$\geq 27 \cdot 5$ & 470 & 36 & 45 & 19 \\
\hline \multicolumn{5}{|l|}{ Waist circumference } \\
\hline $\mathrm{M}<90 \mathrm{~cm} ; \mathrm{F}<80 \mathrm{~cm}$ & 844 & 33 & 44 & 23 \\
\hline$M \geq 90 \mathrm{~cm} ; F \geq 80 \mathrm{~cm}$ & 782 & 44 & 40 & 16 \\
\hline \multicolumn{5}{|l|}{ Waist:hip ratio } \\
\hline$M<0.90 ; F<0.85$ & 971 & 32 & 44 & 24 \\
\hline$M \geq 0.90 ; F \geq 0.85$ & 655 & 46 & 40 & 14 \\
\hline
\end{tabular}

$\mathrm{M}$, males; F, females.

*Some participants ( $n$ 167) did not provide education or income level data, so could not be included in this demographic breakdown.

consumption ( $P$ for trend $<0 \cdot 001)$. Vitamin $C$ intake per $4184 \mathrm{~kJ}$ was lower by $6.5(95 \% \mathrm{CI} 5 \cdot 4,7 \cdot 6) \mathrm{mg}$ in regular fast-food consumers compared with non-consumers.

\section{Dietary guidelines}

At the group level, the mean number of servings of fruit and vegetables consumed daily was below the recommended level for all three groups. At the group level, the mean number of servings of wholegrain consumed daily by non-consumers of fast food was at the recommended level (Table 4). Compared with non-consumers, both regular and occasional consumers of fast food were less likely to meet the wholegrain recommendation of at least one serving daily (Table 5). They were also less likely to consume two servings of fruit daily but were neither more nor less likely to meet the vegetable recommendation of two servings daily. Other factors such as education and income level were associated with meeting these foodbased recommendations (data not shown).
Overall, fast-food consumption status was the strongest predictor of exceeding energy, fat and saturated fat intake recommendations (Table 5). The likelihood of exceeding these recommendations was higher in regular than in occasional consumers. The strongest predictor for exceeding energy intake recommendations was being a regular consumer of fast food (OR $=3 \cdot 89,95 \% \mathrm{CI} 3 \cdot 23,4 \cdot 69)$. Of all dietary recommendations, regular consumers of fast food were most likely to exceed the saturated fat intake recommendation $(\mathrm{OR}=5 \cdot 09,95 \%$ CI 3·89, 6.66).

\section{Weight status}

Occasional consumers of fast food were more likely to have a $\mathrm{BMI} \geq 23 \cdot 0 \mathrm{~kg} / \mathrm{m}^{2}$ than non-consumers $(\mathrm{OR}=1 \cdot 19$, $95 \%$ CI 1.04, 1.37), whereas regular consumers were slightly less likely $(\mathrm{OR}=0 \cdot 76,95 \%$ CI $0 \cdot 64,0 \cdot 91)$ than non-consumers to have an 'at-risk' BMI (Table 5). Fast-food consumers were neither more nor less likely to have a raised waist circumference $(\geq 90 \mathrm{~cm}$ males; $\geq 80 \mathrm{~cm}$ 
Table 2 Weekly frequency of fast-food consumption and daily intake of foods $(\mathrm{g} / \mathrm{d})$ by fast-food consumption status: Singapore residents ( $n$ 1627) aged 18-69 years, 2010 National Nutrition Survey

\begin{tabular}{|c|c|c|c|c|c|c|c|}
\hline & \multicolumn{6}{|c|}{ Fast-food consumption status } & \multirow[b]{3}{*}{$P$ for trend } \\
\hline & \multicolumn{2}{|c|}{$\begin{array}{l}\text { Non-consumer } \\
\quad(n 557)\end{array}$} & \multicolumn{2}{|c|}{$\begin{array}{l}\text { Occasional consumer } \\
\qquad(n 720)\end{array}$} & \multicolumn{2}{|c|}{$\begin{array}{l}\text { Regular consumer } \\
\qquad(n \text { 350) }\end{array}$} & \\
\hline & Mean & SE & Mean & SE & Mean & SE & \\
\hline \multirow[t]{2}{*}{ Weekly consumption frequency of fast food } & $0 \cdot 0$ & $0 \cdot 0$ & $0 \cdot 8$ & $0 \cdot 0$ & $3 \cdot 6$ & $0 \cdot 1$ & - \\
\hline & Median & IQR & Median & IQR & Median & IQR & \\
\hline Total food & 2202 & $1805-2742$ & 2391 & 1900-2919 & 2664 & $2183-3107$ & $<0.001$ \\
\hline Breads and cereals & 69 & $32-199$ & 67 & $37-141$ & 70 & $29-142$ & $<0.001$ \\
\hline Rice and porridge & 444 & $258-584$ & 450 & $297-592$ & 440 & $300-608$ & $<0.001$ \\
\hline Noodles & 212 & $110-382$ & 326 & $189-467$ & 349 & $194-563$ & $<0.001$ \\
\hline Vegetables and beans & 212 & $126-307$ & 226 & $142-292$ & 203 & $130-298$ & 0.001 \\
\hline Fruit & 180 & $89-349$ & 162 & $77-256$ & 173 & $74-272$ & $<0.001$ \\
\hline Poultry & 32 & $14-64$ & 53 & $28-85$ & 60 & $32-98$ & $<0.001$ \\
\hline Meat & 40 & $14-79$ & 54 & $21-94$ & 65 & $30-131$ & $<0.001$ \\
\hline Fish and seafood & 56 & $29-96$ & 60 & $30-100$ & 68 & $30-119$ & $<0.001$ \\
\hline Eggs & 16 & $8-24$ & 20 & $13-32$ & 24 & $15-40$ & $<0.001$ \\
\hline Milk and dairy products & 143 & $3-314$ & 201 & $42-389$ & 163 & $36-309$ & $<0.001$ \\
\hline Desserts, biscuits, and titbits & 54 & $26-108$ & 80 & $42-131$ & 113 & 59-203 & $<0.001$ \\
\hline Fast food & 0 & $0-0$ & 15 & $9-24$ & 63 & $44-88$ & $<0.001$ \\
\hline Sweetened beverages & 10 & $0-43$ & 43 & $15-130$ & 130 & $43-274$ & $<0.001$ \\
\hline Other beverages & 86 & $0-279$ & 43 & $0-200$ & 57 & $0-223$ & $0 \cdot 222$ \\
\hline Soya products & 35 & $13-82$ & 33 & $13-70$ & 55 & $15-125$ & $<0.001$ \\
\hline Miscellaneoust & 66 & $20-231$ & 71 & 27-191 & 75 & $28-215$ & $<0.001$ \\
\hline
\end{tabular}

IQR, interquartile range.

*Variables were log-transformed before using linear contrasts to test for trends

tMiscellaneous includes items such as bread spreads, salad dressings and soup broths.

Table 3 Daily intakes of nutrients (total and per $4184 \mathrm{~kJ}(1000 \mathrm{kcal}))$ by fast-food consumption status, adjusted for age, gender, ethnicity, education and income level: Singapore residents ( $n$ 1627) aged 18-69 years, 2010 National Nutrition Survey

\begin{tabular}{|c|c|c|c|c|c|c|c|}
\hline & \multicolumn{6}{|c|}{ Fast-food consumption status } & \multirow[b]{3}{*}{$P$ for trend } \\
\hline & \multicolumn{2}{|c|}{$\begin{array}{l}\text { Non-consumer } \\
\quad(n 557)\end{array}$} & \multicolumn{2}{|c|}{$\begin{array}{c}\text { Occasional consumer } \\
(n \text { 720) }\end{array}$} & \multicolumn{2}{|c|}{$\begin{array}{l}\text { Regular consumer } \\
\text { ( } n \text { 350) }\end{array}$} & \\
\hline & Mean & SE & Mean & SE & Mean & SE & \\
\hline Energy (kJ) & 10234 & $39 \cdot 62$ & 11175 & $34 \cdot 52$ & 11941 & $67 \cdot 20$ & $<0.001$ \\
\hline Energy (kcal) & 2446 & $9 \cdot 47$ & 2671 & 8.25 & 2854 & $16 \cdot 06$ & $<0.001$ \\
\hline Carbohydrate (g) & 320 & $1 \cdot 17$ & 341 & 1.03 & 362 & $1 \cdot 91$ & $<0.001$ \\
\hline Carbohydrate (g/4184 kJ) & 133 & $0 \cdot 18$ & 129 & $0 \cdot 11$ & 128 & $0 \cdot 17$ & $<0.001$ \\
\hline Protein $(g)$ & 93 & $0 \cdot 38$ & 102 & $0 \cdot 33$ & 109 & 0.63 & $<0.001$ \\
\hline Protein (g/4184 kJ) & 38 & 0.07 & 38 & 0.06 & 38 & 0.09 & 0.627 \\
\hline Total fat $(\mathrm{g})$ & 84 & 0.38 & 96 & 0.34 & 104 & 0.72 & $<0.001$ \\
\hline Total fat $(\mathrm{g} / 4184 \mathrm{~kJ})$ & 34 & 0.06 & 35 & 0.04 & 36 & 0.06 & $<0.001$ \\
\hline $\operatorname{SFA}(g)$ & 31 & $0 \cdot 15$ & 36 & $0 \cdot 14$ & 40 & 0.30 & $<0.001$ \\
\hline SFA (g/4184kJ) & 13 & 0.03 & 13 & 0.02 & 14 & 0.03 & $<0.001$ \\
\hline MUFA $(g)$ & 31 & $0 \cdot 15$ & 36 & $0 \cdot 14$ & 39 & $0 \cdot 27$ & $<0.001$ \\
\hline MUFA (g/4184 kJ) & 12 & 0.03 & 13 & 0.02 & 13 & 0.03 & $<0.001$ \\
\hline PUFA (g) & 15 & 0.07 & 17 & 0.05 & 19 & $0 \cdot 12$ & $<0.001$ \\
\hline PUFA (g/4184 kJ) & 6 & 0.02 & 6 & 0.01 & 6 & 0.02 & $<0.001$ \\
\hline Trans fat $(\mathrm{g})$ & 0 & 0.00 & 0 & 0.00 & 1 & 0.00 & $<0.001$ \\
\hline Trans fat $(\mathrm{g} / 4184 \mathrm{~kJ})$ & 0 & 0.00 & 0 & 0.00 & 0 & 0.00 & $<0.001$ \\
\hline Cholesterol (mg) & 305 & $1 \cdot 77$ & 353 & 1.65 & 382 & $2 \cdot 70$ & $<0.001$ \\
\hline Cholesterol (mg/4184 kJ) & 122 & 0.51 & 130 & $0 \cdot 37$ & 132 & 0.50 & $<0.001$ \\
\hline Dietary fibre $(\mathrm{g})$ & 23 & 0.09 & 24 & 0.07 & 25 & $0 \cdot 12$ & $<0.001$ \\
\hline Dietary fibre (g/4184 kJ) & 10 & 0.03 & 9 & 0.02 & 9 & 0.03 & $<0.001$ \\
\hline Vitamin $A(\mu g)$ & 941 & 3.90 & 996 & $2 \cdot 70$ & 1011 & $4 \cdot 86$ & $<0.001$ \\
\hline Vitamin A ( $\mu \mathrm{g} / 4184 \mathrm{~kJ})$ & 400 & $1 \cdot 71$ & 388 & $1 \cdot 12$ & 369 & $1 \cdot 79$ & $<0.001$ \\
\hline Vitamin C (mg) & 128 & 0.68 & 134 & 0.42 & 131 & 0.60 & 0.004 \\
\hline Vitamin C (mg/4184 kJ) & 55 & 0.33 & 53 & 0.22 & 49 & 0.32 & $<0.001$ \\
\hline $\mathrm{Ca}(\mathrm{mg})$ & 755 & 3.94 & 809 & $2 \cdot 44$ & 831 & $4 \cdot 65$ & $<0.001$ \\
\hline $\mathrm{Ca}(\mathrm{mg} / 4184 \mathrm{~kJ})$ & 318 & $1 \cdot 70$ & 312 & 1.07 & 299 & $1 \cdot 30$ & $<0.001$ \\
\hline $\mathrm{Fe}(\mathrm{mg})$ & 17 & 0.06 & 18 & 0.05 & 19 & 0.09 & $<0.001$ \\
\hline $\mathrm{Fe}(\mathrm{mg} / 4184 \mathrm{~kJ})$ & 7 & 0.02 & 7 & 0.01 & 7 & 0.02 & $<0.001$ \\
\hline
\end{tabular}


Table 4 Daily intakes of selected foods and nutrients in comparison with dietary recommendations, and percentage contribution of fast food to daily intakes of energy and selected nutrients, by fast-food consumption status: Singapore residents ( $n$ 1627) aged 18-69 years, 2010 National Nutrition Survey

\begin{tabular}{|c|c|c|c|c|c|c|c|c|}
\hline & \multirow[b]{3}{*}{ Recommendation } & \multicolumn{6}{|c|}{ Fast-food consumption status } & \multirow[b]{3}{*}{$P$ for trend } \\
\hline & & \multicolumn{2}{|c|}{$\begin{array}{l}\text { Non-consumer } \\
\quad(n 557)\end{array}$} & \multicolumn{2}{|c|}{$\begin{array}{l}\text { Occasional consumer } \\
\qquad(n 720)\end{array}$} & \multicolumn{2}{|c|}{$\begin{array}{l}\text { Regular consumer } \\
\qquad(n \text { 350) }\end{array}$} & \\
\hline & & Mean & SE & Mean & $\mathrm{SE}$ & Mean & SE & \\
\hline Wholegrain intake (servings/d) & 1 & 1.00 & 0.03 & 0.63 & 0.02 & 0.59 & 0.03 & $<0.001$ \\
\hline Fruit intake (servings/d) & 2 & 1.43 & 0.02 & $1 \cdot 20$ & 0.02 & $1 \cdot 11$ & 0.02 & $<0.001$ \\
\hline Vegetable intake (servings/d) & 2 & $1 \cdot 71$ & 0.02 & 1.79 & 0.02 & $1 \cdot 90$ & 0.03 & $<0.001$ \\
\hline Energy intake* $(\%$ of RDA) & $M \leq 10857 \mathrm{~kJ} / \mathrm{d}$ & $103 \cdot 72$ & 0.74 & $114 \cdot 58$ & 0.67 & $130 \cdot 42$ & $1 \cdot 04$ & $<0.001$ \\
\hline Equivalent daily contribution of fast food to energy intake (\%) & $\begin{array}{l}(2595 \mathrm{kcal} / \mathrm{d}) \\
\mathrm{F} \leq 8527 \mathrm{~kJ} / \mathrm{d} \\
(2038 \mathrm{kcal} / \mathrm{d})\end{array}$ & 0.00 & 0 & $1 \cdot 72$ & 0.02 & 6.09 & $0 \cdot 10$ & $<0.001$ \\
\hline Total fat intake (\% of total energy) & $<30 \%$ & $29 \cdot 55$ & $0 \cdot 10$ & $31 \cdot 93$ & 0.09 & $33 \cdot 50$ & $0 \cdot 11$ & $<0.001$ \\
\hline Equivalent daily contribution of fast food to total fat intake (\%) & & 0.00 & 0 & $2 \cdot 31$ & 0.03 & $7 \cdot 89$ & $0 \cdot 13$ & $<0.001$ \\
\hline SFA intake (\% of total energy) & $<10 \%$ & $10 \cdot 85$ & 0.05 & $12 \cdot 11$ & 0.04 & 12.94 & 0.06 & $<0.001$ \\
\hline Equivalent daily contribution of fast food to SFA intake (\%) & & 0.00 & 0 & $2 \cdot 23$ & 0.03 & $7 \cdot 38$ & $0 \cdot 12$ & $<0.001$ \\
\hline Weekly consumption frequency of fast food & & 0.00 & 0 & 0.8 & 0.01 & $3 \cdot 6$ & 0.06 & $<0.001$ \\
\hline
\end{tabular}

M, males; F, females.

${ }^{*}$ Mean energy intake recommendations; age- and gender-specific recommendations are used in all calculations.

Table 5 The odds of regular and occasional fast-food consumers meeting or exceeding dietary intake recommendations, and of having an 'at-risk' BMI and abdominal obesity: Singapore residents ( $n$ 1460) aged 18-69 years, 2010 National Nutrition Survey

\begin{tabular}{|c|c|c|c|c|c|c|c|c|c|c|c|c|}
\hline \multirow[b]{3}{*}{ Dependent variable } & \multicolumn{6}{|c|}{ Unadjusted } & \multicolumn{6}{|c|}{ Adjusted* } \\
\hline & \multicolumn{3}{|c|}{ Occasional consumer } & \multicolumn{3}{|c|}{ Regular consumer } & \multicolumn{3}{|c|}{ Occasional consumer } & \multicolumn{3}{|c|}{ Regular consumer } \\
\hline & ORt & $95 \% \mathrm{Cl}$ & $P$ value & ORt & $95 \% \mathrm{Cl}$ & $P$ value & ORt & $95 \% \mathrm{Cl}$ & $P$ value & ORt & $95 \% \mathrm{Cl}$ & $P$ value \\
\hline Wholegrain intake $\geq 1$ serving $/ \mathrm{d}$ & 0.57 & $0.50,0.64$ & $<0.001$ & 0.45 & $0.38,0.53$ & $<0.001$ & 0.58 & $0.50,0.67$ & $<0.001$ & 0.53 & $0.43,0.64$ & $<0.001$ \\
\hline Fruit intake $\geq 2$ servings $/ d$ & 0.52 & $0.46,0.59$ & $<0.001$ & 0.45 & $0.38,0.53$ & $<0.001$ & 0.43 & $0.37,0.50$ & $<0.001$ & 0.46 & $0.37,0.55$ & $<0.001$ \\
\hline Vegetable intake $\geq 2$ servings/d & $1 \cdot 17$ & $1 \cdot 04,1.32$ & 0.009 & $1 \cdot 51$ & $1 \cdot 31,1 \cdot 74$ & $<0.001$ & 1.08 & $0.93,1.24$ & $0 \cdot 327$ & $1 \cdot 19$ & $0.99,1.42$ & 0.062 \\
\hline Energy intake $\geq \mathrm{RDA}$ & $1 \cdot 71$ & $1.53,1.91$ & $<0.001$ & $3 \cdot 66$ & $3 \cdot 14,4 \cdot 27$ & $<0.001$ & 1.85 & $1 \cdot 61,2 \cdot 11$ & $<0.001$ & 3.89 & $3 \cdot 23,4 \cdot 69$ & $<0.001$ \\
\hline Fat intake $\geq$ RDA & $2 \cdot 80$ & $2 \cdot 49,3 \cdot 14$ & $<0.001$ & $5 \cdot 24$ & $4 \cdot 45,6 \cdot 18$ & $<0.001$ & $2 \cdot 12$ & $1 \cdot 85,2 \cdot 44$ & $<0.001$ & $3 \cdot 60$ & $2 \cdot 96,4 \cdot 38$ & $<0.001$ \\
\hline Saturated fat intake $\geq R D A$ & $3 \cdot 16$ & $2 \cdot 76,3 \cdot 62$ & $<0.001$ & $6 \cdot 60$ & $5 \cdot 27,8 \cdot 26$ & $<0.001$ & $2 \cdot 34$ & $1 \cdot 99,2 \cdot 76$ & $<0.001$ & $5 \cdot 09$ & $3 \cdot 89,6 \cdot 66$ & $<0.001$ \\
\hline $\mathrm{BMI} \geq 23 \mathrm{~kg} / \mathrm{m}^{2}$ & 1.07 & $0.96,1.20$ & 0.23 & 0.69 & $0.60,0.79$ & $<0.001$ & $1 \cdot 19$ & $1 \cdot 04,1 \cdot 37$ & 0.014 & 0.76 & $0.64,0.91$ & 0.003 \\
\hline$W C, M \geq 90 \mathrm{~cm}, F \geq 80 \mathrm{~cm}$ & 0.68 & $0.61,0.76$ & $<0.001$ & 0.51 & $0.44,0.59$ & $<0.001$ & 1.07 & $0.93,1.23$ & 0.333 & 0.98 & $0 \cdot 82,1 \cdot 17$ & 0.793 \\
\hline WHR, $M \geq 0.90, F \geq 0.85$ & 0.63 & $0.56,0.71$ & $<0.001$ & $0 \cdot 40$ & $0.34,0.46$ & $<0.001$ & $1 \cdot 52$ & $1 \cdot 32,1 \cdot 77$ & $<0.001$ & $1 \cdot 24$ & $1 \cdot 03,1.51$ & 0.027 \\
\hline
\end{tabular}


females) than non-consumers, but were more likely to have a raised waist:hip ratio above the WHO cut-off ( $\geq 0.90$ males; $\geq 0.85$ females). Overall, ethnicity was the strongest predictor of weight status (data not shown).

\section{Discussion}

The present analysis has shown that fast-food consumption frequency is associated with declining dietary quality and that one in five adult Singapore residents consumes fast food at least once per week. In line with other studies, the analysis showed that frequency of fast-food consumption is associated with exceeding recommendations for energy ${ }^{(12,29)}$, fat and saturated fat intakes ${ }^{(10)}$ and with not meeting recommendations for wholegrain $^{(9)}$ and fruit consumption ${ }^{(9-12)}$.

Fast-food consumption was most prevalent in young adults, high income level groups and middle education level groups. These demographic findings have also been observed overseas ${ }^{(10,11,18-20,30)}$ and reflect the target market of the fast-food industry, especially in $\mathrm{Asia}^{(31)}$.

Although frequency of consumption is similar to that in the USA, the motivators of use may not be. In the USA, speed ${ }^{(32)}$ and convenience ${ }^{(18)}$ have been identified as the strongest motivators for choosing fast food; in Asia other motivators have been identified, such as fast food's fashionable status, association with Western culture ${ }^{(5)}$ and advertising $^{(7)}$. The industry has also identified that young consumers are attracted by a clean, comfortable environment in which they can socialise ${ }^{(33)}$. Thai adolescents were asked about reasons for fast-food consumption; the association with a modern lifestyle, the venue being desirable for social occasions and advertising emerged as important factors $^{(7)}$. In recent years McDonalds and KFC in Singapore have been among the top ten for media advertisement expenditure $^{(31)}$ and in a recent market research survey McDonalds and KFC were ranked third and eighth respectively as favourite brands of Singaporean adolescents ${ }^{(34)}$.

In the present study frequency of fast-food consumption is associated with higher intakes of food in total, and this is across most food groups. This is in line with the findings of a local cohort study in older Chinese Singaporeans ${ }^{(17)}$ which suggest that fast-food consumption is not a marker for an overall dietary pattern defined by food type. The higher intake of most foods suggests that fast-food consumption reflects wider eating behaviours and is not the sole cause of higher energy intakes. In fact, it has been suggested that excessive consumption of these fast foods is likely to reflect excessive consumption of other foods and excessive consumption in other lifestyle choices such as purchasing in shops and supermarkets $^{(35)}$.

The present results show that occasional fast-food consumers are the most likely to have a raised BMI, whereas regular fast-food consumers are less likely. This is in contrast to the energy intake data, where the regular fast-food consumers have a higher energy intake than both occasional and non-consumers. One possible reason is that participants with higher BMI under-reported their intake of fast food compared with participants of normal body weight. Overweight and obese individuals have been shown to under-report their energy intake ${ }^{(36)}$, and under-report foods perceived as "unhealthy, (37), so it is possible that those with higher BMI have not reported as accurately as those with lower BMI. Conversely, it could be that overweight and obese participants avoid fast foods in an attempt to restrict energy intake. Since the data are cross-sectional it is not possible to infer the direction of any associations. Physical activity levels were not included in the analysis; energy RDA are based on a population-level average physical activity level of 1.61 based on data obtained in the NHS; however, activity levels varied by demographics ${ }^{(1)}$, so use of an average physical activity level may have some impact on the estimates of proportions exceeding energy recommendations, although the direction of this impact is unknown. Another possible explanation is that since weight gain occurs as a result of long-term positive energy balance, and most fast-food consumers were in the younger age groups, they have not yet been in positive energy balance long enough to exceed a BMI of $23 \cdot 0 \mathrm{~kg} / \mathrm{m}^{2}$. However fast-food consumers are more likely to have a raised waist:hip ratio than non-consumers. While waist:hip ratio can identify abdominal fatness, it may not identify overweight and obese individuals who have high hip circumferences. This suggests that fast-food consumption frequency may be associated with abdominal fatness, rather than overall overweight/obesity. Despite these findings on BMI, any group habitually exceeding energy intake recommendations is at risk of being in positive energy balance. Overweight and obesity are caused by energy imbalances over time, i.e. energy intake persistently exceeding energy expenditure, and weight gain over a 5- or 10-year period has been shown to occur at an imbalance of less than $418 \mathrm{~kJ} / \mathrm{d}(100 \mathrm{kcal} / \mathrm{d})^{(38)}$.

The present study has several strengths. First, the data are from an ethnically diverse, nationally representative sample. There are no other similar data in Singapore on habitual nutrient intakes at the population level. Second, diet is assessed using an FFQ, which has been suggested as the most appropriate tool for measuring habitual fast-food consumption $^{(39)}$. However, it must be noted that the FFQ remains a self-report tool prone to biases such as misreporting, the distribution of which is unknown in this sample, and the portion sizes assigned to each item are largely averages; this may have impacted the calculation of nutrient intakes. In general, FFQ have a tendency to overestimate dietary intake ${ }^{(40)}$ and the cognitive challenge of an FFQ may be greater in individuals with a highly varied diet.

In the literature, there is variation in the definition of fast food. Should it refer to the food type, or the nature of the outlet it is purchased from? For example, should a 
salad from a burger outlet be classified as fast food? In the present study the definition was based on participant perception of fast food - if the interest is food type, this approach may have resulted in an underestimate of intake frequency, since there are a number of smaller local chains serving similar types of food which may not have been perceived as fast food because of the brand. Another factor which may have resulted in underestimation of fast-food intake is the limited number of items in the fast-food section of the FFQ. For example, fried chicken was not included in this list of fast-food items since the FFQ did not ask about the venue in which these foods were consumed, and fried chicken is a popular ethnic dish frequently consumed elsewhere.

In order to curb the impact of fast-food consumption, it has been suggested that energy (calorie) labelling of fast-food menus would affect consumer choices, and although this has been shown to increase awareness of energy content ${ }^{(41)}$, there is little or no impact on subsequent energy intake ${ }^{(42)}$. However, a recent study has shown that $14-33 \%$ of consumers invited to down-size their portions in a fast-food outlet at the point of purchase accepted this offer and were served more than $837 \mathrm{~kJ}$ $(200 \mathrm{kcal})$ less than they initially ordered ${ }^{(43)}$. Down-sizing did not impact the amount of leftover food, suggesting that energy intake was also reduced as a result. In the Asian setting, where fast-food outlets are viewed as a desirable place to socialise, ensuring that alternative venues, equally as clean and comfortable, are available has been suggested as an important measure ${ }^{(7)}$, although it is likely that considerable social marketing would be required to shift trends away from established venues of choice towards healthier alternatives.

\section{Conclusion}

Fast-food consumption in Singapore is most prevalent in young adults, high income and middle education level groups. Frequent fast-food consumption is associated with unfavourable dietary and nutrient profiles and abdominal obesity, although associated with having an 'at-risk' BMI only in occasional consumers. Overconsumption of food, especially energy-dense, nutrient-poor foods, is not recommended. Measures are required to empower consumers to make healthier choices in all settings; this includes efforts from industry to control portion sizes and more heavily market items which are less energy-dense. The Health Promotion Board will continue with efforts to engage all sectors of the food service industry in order that Singaporeans have access to healthier food choices.

\section{Acknowledgements}

Sources of funding: This study was funded by the Health Promotion Board. Conflicts of interest: No author declares a conflict of interest. Authors' contributions: C.W. prepared the manuscript and conducted analyses. Y.M. prepared the data set, provided input and reviewed the manuscript. A.C.B. provided input and reviewed the manuscript. M.F.C. was Principal Investigator for the survey and provided input and reviewed the manuscript. L.C. provided input and reviewed the manuscript. Acknowledgements: Anita Lai is acknowledged for planning and project managing the survey and creating the nutrient table required for FFQ analysis.

\section{References}

1. Ministry of Health, Epidemiology and Disease Control Division (2011) National Health Survey 2010. Singapore: Ministry of Health Singapore; available at http:// www.moh.gov.sg/content/moh_web/home/Publications/ Reports/2011/national_health_survey2010.html

2. Health Promotion Board (2013) Report of the National Nutrition Survey 2010. Singapore: Health Promotion Board; available at http://www.hpb.gov.sg/HOPPortal/content/ conn/HOPUCM/path/Contribution\%20Folders/uploaded Files/HPB_Online/Publications/NNS-2010.pdf

3. Ministry of Trade and Industry, Department of Statistics (2009) Report on the Household Expenditure Survey 2007/08. http:// www.singstat.gov.sg/publications/publications_and_papers/ household_income_and_expenditure/hes2007.pdf (accessed December 2012).

4. Ministry of Trade and Industry, Department of Statistics (2010) Economic surveys series 2010: Food and Beverage services, Reference Year 2010. http://www.singstat.gov.sg/ pubn/business/essfnb2010.pdf (accessed June 2012).

5. Cheng TO (2006) Effects of fast foods, rising blood pressure and increasing serum cholesterol on cardiovascular disease in China. Am J Cardiol 97, 1676-1678.

6. Noor MI (2002) The nutrition and health transition in Malaysia. Public Health Nutr 5, 191-195.

7. Seubsman S, Kelly M, Yuthapornpinit P et al. (2009) Cultural resistance to fast-food consumption? A study of youth in north eastern Thailand. Int J Consum Stud 33, 669-675.

8. Prentice AM \& Jebb SA (2003) Fast foods, energy density and obesity: a possible mechanistic link. Obes Rev $\mathbf{4}$, 187-194.

9. Pereira MA, Kartashov AI, Ebbeling CB et al. (2005) Fastfood habits, weight gain, and insulin resistance (the CARDIA study): 15-year prospective analysis. Lancet $\mathbf{3 6 5}$, 36-42.

10. Paeratakul S, Ferdinand DP, Champagne CM et al. (2003) Fast-food consumption among US adults and children: dietary and nutrient intake profile. J Am Diet Assoc 103, $1332-1338$.

11. Schroder H, Fito M \& Covas MI (2007) Association of fast food consumption with energy intake, diet quality, body mass index and the risk of obesity in a representative Mediterranean population. Br J Nutr 98, 1274-1280.

12. French SA, Harnack L \& Jeffery RW (2000) Fast food restaurant use among women in the Pound of Prevention study: dietary, behavioral and demographic correlates. Int J Obes Relat Metab Disord 24, 1353-1359.

13. Jeffery RW, Baxter J, McGuire M et al. (2006) Are fast food restaurants an environmental risk factor for obesity? Int $J$ Behav Nutr Phys Act 3, 2.

14. Li M, Dibley MJ, Sibbritt DW et al. (2010) Dietary habits and overweight/obesity in adolescents in Xi'an City, China. Asia Pac J Clin Nutr 19, 76-82. 
15. Bes-Rastrollo M, Sanchez-Villegas A, Gomez-Gracia E et al. (2006) Predictors of weight gain in a Mediterranean cohort: the Seguimiento Universidad de Navarra Study 1. Am J Clin Nutr 83, 362-370.

16. Duffey KJ, Gordon-Larsen P, Jacobs DR Jr et al. (2007) Differential associations of fast food and restaurant food consumption with 3-y change in body mass index: the Coronary Artery Risk Development in Young Adults Study. Am J Clin Nutr 85, 201-208.

17. Odegaard AO, Woon PK, Yuan J-M et al. (2012) Westernstyle fast food intake and cardio-metabolic risk in an eastern country. Circulation 126, 182-188.

18. Dave JM, An LC, Jeffery RW et al. (2009) Relationship of attitudes toward fast food and frequency of fast-food intake in adults. Obesity (Silver Spring) 17, 1164-1170.

19. Mohr P, Wilson C, Dunn K et al. (2007) Personal and lifestyle characteristics predictive of the consumption of fast foods in Australia. Public Health Nutr 10, 1456-1463.

20. Steyn NP, Labadarios D \& Nel JH (2011) Factors which influence the consumption of street foods and fast foods in South Africa - a national survey. Nutr J 10, 104.

21. World Health Organization (1990) MONICA Manual. Geneva: WHO; available at http://www.ktl.fi/publications/ monica/manual/index.htm

22. WHO Expert Consultation (2004) Appropriate body-mass index for Asian populations and its implications for policy and intervention strategies. Lancet 363, 157-163.

23. World Health Organization (2011) Waist Circumference and Waist-Hip Ratio: Report of a WHO Expert Consultation, Geneva, 8-11 December 2008. Geneva: WHO; available at http://whqlibdoc.who.int/publications/2011/ 9789241501491_eng.pdf

24. Deurenberg-Yap M, Li T, Tan WL et al. (2000) Validation of a semi-quantitative food frequency questionnaire for estimation of intakes of energy, fats and cholesterol among Singapore. Asia Pac J Clin Nutr 9, 282-288.

25. Moore LV, Diez Roux AV, Nettleton JA et al. (2009) Fast-food consumption, diet quality, and neighborhood exposure to fast food: the multi-ethnic study of atherosclerosis. Am J Epidemiol 170, 29-36.

26. Scully M, Dixon H \& Wakefield M (2009) Association between commercial television exposure and fast-food consumption among adults. Public Health Nutr 12, 105-110.

27. Health Promotion Board (2012) Food-based dietary guidelines for adults. http://www.hpb.gov.sg/HOPPortal/article?id=2758 (accessed December 2012).

28. Henry CJ (2005) Basal metabolic rate studies in humans: measurement and development of new equations. Public Health Nutr 8, 1133-1152.

29. Jeffery RW \& French SA (1998) Epidemic obesity in the United States: are fast foods and television viewing contributing? Am J Public Health 88, 277-280.
30. Hamer M \& Mishra GD (2010) Dietary patterns and cardiovascular risk markers in the UK Low Income Diet and Nutrition Survey. Nutr Metab Cardiovasc Dis 20, 491-497.

31. Hawkes C (2002) Marketing activities of global soft drink and fast food companies in emerging markets: a review. In Globalization, Diets and Noncommunicable Diseases. Geneva: WHO; available at http://whqlibdoc.who.int/ publications/9241590416.pdf

32. Rydell SA, Harnack LJ, Oakes JM et al. (2008) Why eat at fast-food restaurants: reported reasons among frequent consumers. J Am Diet Assoc 108, 2066-2070.

33. Euromonitor International (2012) Southeast Asia's importance in consumer foodservice. http://blog.euromonitor.com/2012/ 06/southeast-asias-importance-in-consumer-foodservice.html (accessed December 2012).

34. TNS (2011) The TNS TRU Singapore Teen Study 2011. Singapore: TNS Singapore.

35. Simmons D, McKenzie A, Eaton S et al. (2005) Choice and availability of takeaway and restaurant food is not related to the prevalence of adult obesity in rural communities in Australia. Int J Obes (Lond) 29, 703-710.

36. Rennie KL, Coward A \& Jebb SA (2007) Estimating underreporting of energy intake in dietary surveys using an individualised method. Br J Nutr 97, 1169-1176.

37. Pryer JA, Vrijheid M, Nichols R et al. (1997) Who are the 'low energy reporters' in the dietary and nutritional survey of British adults? Int J Epidemiol. 1997 26, $146-154$

38. Hill JO (2009) Can a small-changes approach help address the obesity epidemic? A report of the Joint Task Force of the American Society for Nutrition, Institute of Food Technologists, and International Food Information Council. Am J Clin Nutr 89, 477-484.

39. Rosenheck R (2008) Fast food consumption and increased caloric intake: a systematic review of a trajectory towards weight gain and obesity risk. Obes Rev 9, 535-547.

40. Bingham SA, Gill C, Welch A et al. (1994) Comparison of dietary assessment methods in nutritional epidemiology: weighed records v. $24 \mathrm{~h}$ recalls, food-frequency questionnaires and estimated-diet records. Br J Nutr 72, 619-643.

41. Dumanovsky T, Huang CY, Bassett MT et al. (2010) Consumer awareness of fast-food calorie information in New York City after implementation of a menu labeling regulation. Am J Public Health 100, 2520-2525.

42. Swartz JJ, Braxton D \& Viera AJ (2011) Calorie menu labeling on quick-service restaurant menus: an updated systematic review of the literature. Int J Behav Nutr Phys Act 8, 135.

43. Schwartz J, Riis J, Elbel B et al. (2012) Inviting consumers to downsize fast-food portions significantly reduces calorie consumption. Health Aff (Millwood) 31, 399-407. 